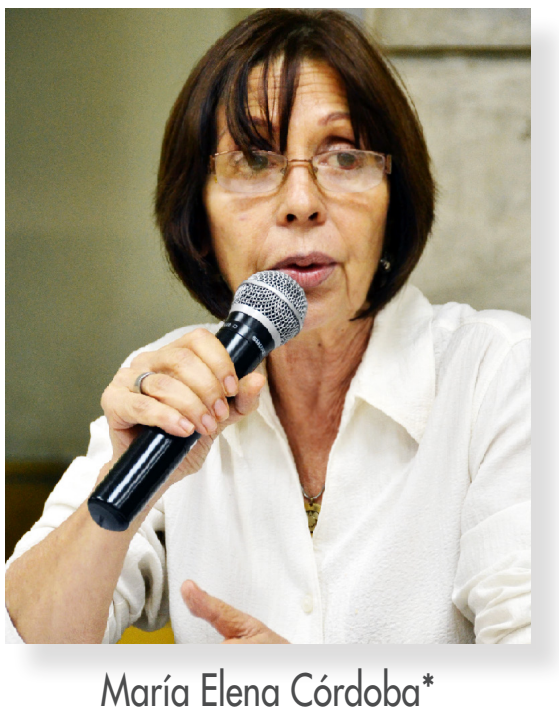

\title{
Diseño curricular basado en competencias en la educación superior
}

\section{Competency-based curriculum design in higher education}

Recibido: 27-03-17

Aprobado: 01-06-17

\begin{abstract}
"Si me caí, es porque estaba caminando. Y caminar vale la pena, aunque te caigas." Eduardo Galeano
\end{abstract}

\section{Resumen}

El interés principal de este artículo consistió en abordar algunas de las implicaciones del controversial tema de las competencias y su inclusión en el currículo. Parte de la comprensión de que, así como hay diversas teorías sobre educación, así también existen distintas modalidades e interpretaciones respecto al currículo y las competencias. Este enfoque que se viene poniendo en práctica desde hace ya varios años a nivel mundial, demanda a las instituciones educativas estándares de calidad, replanteo del proceso de evaluación, articulación de todas las instancias institucionales en función de esta forma de trabajo y posicionamiento de la acepción de competencias a asumir, ya que algunas acepciones asumidas implican un retroceso, otras un estancamiento y hay algunas que impulsan prácticas realmente innovadoras, susceptibles de mejorar sustancialmente la educación. Por otra parte, hay que considerar que estamos en una época de grandes cambios, en que la complejidad del mundo actual exige a las universidades que eduquen para el cambio y la incertidumbre. Hacerlo desde una concepción de competencias implica una serie de compromisos, replanteos y modificaciones en toda su estructura, para lo cual varias de las universidades que en teoría definen su currículo por competencias, no están preparadas para hacerlo. Los espacios de reflexión participativa, la inclusión de todos los actores en esta redefinición curricular podría acercarnos a planteos más contextualizados y realistas.

\section{Abstract}

This article tackles some of the implications of a competency-based curriculum. There is great controversy around the competency-based curriculum in part because of the different interpretations educational institutions attach to it. This approach, that has been implemented for a number of years worldwide, demands from institutions, among other elements, quality standards, revision of assessment processes, and the articulation of the institution as a whole in terms of ways of working. Another important implication is the decision of which competencies should be included and which not since some imply moving backwards or not advancing at all. However, other competencies promote innovative practices and are susceptible to improve education. On the other hand, it is important to consider that we are in an era of great changes, in that the complexity of today's world requires universities that educate for change and uncertainty. To approach this situation from the conception of competence involves a series of commitments, redesigns and modifications in the institution structure as a whole, for which several universities that, in theory, define their curricula as competency-based, are not prepared to do so. Spaces of participatory reflection, the inclusion of all stakeholders in this curriculum redefinition could bring us closer to more contextualized and realistic scenarios.

\section{Palabras clave}

currículo; competencias; prácticas innovadoras; incertidumbre; reflexión participativa

\section{Keywords}

curriculum; competencies; innovative practices; uncertainty; participatory reflection

*María Elena Córdoba: Actualmente se desempeña como Docente Investigadora en el Instituto Tecnológico de Santo Domingo, y consultora independiente en materia educativa, con énfasis en los campos de Estudios Generales, transdisciplinariedad, currículo y evaluación auténtica. Cursó Psicología, Educación Social, Animación Sociocultural y Doctorado en Educación. Para contactar a la autora: macorbu@yahoo.com 
Abordar un tema del que tanto se ha dicho y del que se tienen posturas tan diferentes, resulta complejo, así como existen numerosas teorías sobre educación, también encontramos distintas modalidades e interpretaciones respecto al currículo y las competencias. Esta época de grandes cambios, exige a las universidades educar para el cambio y la incertidumbre. Se requiere analizar, que la formación en una carrera o disciplina, no será suficiente para que quienes la adquieran puedan adaptarse a los grandes cambios que se avecinan (López, Sena \& Javier 2012).

Educar para el cambio y la incertidumbre requiere que las instituciones de educación superior reconozcan y asuman la relevancia de una educación integral que prepare al futuro profesional en su dimensión psicobiosocial a través de un curriculum capaz de dar respuesta a la complejidad del mundo y las necesidades de la sociedad. Además, es importante que se identifiquen, analicen y superen las discrepancias entre el currículo como documento y la representación del currículo que los docentes hacen dentro de los salones de clase.

No es suficiente reconocer tales discrepancias, hay que construir diseños más realistas, contextualizados y en los que el cuerpo docente sea parte del mismo.

Es probable que un alto número de profesionales en diseño curricular coincidan en que: el currículo tiene que entenderse como un campo especialmente abonado para la investigación y cuyos resultados deben concretarse en la práctica, así como que, en su construcción, se atiende a una propuesta eminentemente técnica. Sin embargo, en lo que quizá no todas las opiniones converjan, es que tal construcción incluye subjetividades. Pinar (2014), un referente sin duda en materia curricular cuyos aportes han removido las estructuras tradicionales de su abordaje, comienza la etapa de lo que se llama la reconceptualización curricular; en la que plantea el currículo como un camino, que denomina currere, un recorrido en el que los individuos se reconstruyen reconociendo su realidad. Detrás de cada propuesta curricular hay una historia, cargada de subjetividades, no se parte de cero. Además de que cada currículo refleja evidentemente un punto de vista institucional, tanto en la visión macro que se materializa en el proyecto curricular, como en la práctica, que se materializa en las adecuaciones al currículo que los docentes hacen en sus clases.

Para que las universidades logren encaminar el cambio social a partir de la reconstrucción del currículo es necesario que, tanto estudiantes como docentes participen activamente en ser orientadores de su propio currere. De esta forma, por ejemplo, los estudiantes se convierten en generadores de su autoestudio y del proceso de metacognición.

En el caso particular de diseños por competencias, es necesario considerar que las competencias académicas avalan los avances para el dominio de las disciplinas, y evidentemente que son muy importantes, sin embargo, la persona que desarrollará las competencias de esa disciplina está viviendo en sociedad, con un cuerpo físico, una mente, un espíritu, emociones, creencias, ilusiones, familia, amigos, etc.

Este ser humano será competente si es capaz de desarrollarunaformaciónintegral, concompetenciaspara la vida, que incorpora ese conglomerado de contexto, en el proceso de reconstrucción social. Este es el reto que deben asumir las universidades en el diseño de su oferta académica.

\section{Currículo por competencias}

El que hoy en día se pueda estudiar en cualquier momento y en cualquier lugar, que la virtualización ha ganado espacio más rápido de lo que como universidades podíamos alcanzar; que exista tanta variedad de profesiones y la necesidad de resolver problemas en un entorno cambiante y complejo, son algunas de las razones que favorecen, en los defensores de esta propuesta, la idea de que las competencias podrían constituir la solución a un aprendizaje que genera respuestas, que está a tono con la complejidad del mundo actual y que ofrece mejores resultados para la diversificación de la oferta laboral.

"El concepto de competencia conecta de alguna manera con preocupaciones reales y con intuiciones fuertemente arraigadas entre los responsables de las políticas educativas y los profesionales de la educación, sobre cómo afrontar los problemas y desafíos actuales de la educación escolar"

(Coll, 2007 citado en Coll, 2009, p.9).

Este enfoque que se viene poniendo en práctica desde hace ya varios años a nivel mundial, demanda de las universidades estándares de calidad, procesos de evaluación, definición de competencias y posicionamiento de la acepción a asumir, porque no es posible pensar en la formación integral, cuando en múltiples acepciones de competencias, el propósito es que la persona sea competente para desempeñarse ante determinadas situaciones, con un aprendizaje orientado fundamentalmente a la práctica profesional. 
Se hace evidente la necesidad de constituir diseños que consideren al ser humano en sociedad, inserto en el ambiente, como parte de él, considerando las competencias necesarias para la vida cotidiana, con vista al desarrollo de ciudadanos responsables comprometidos con su propio bienestar y el bien común. Un currículo por competencias, demanda partir de la evaluación del perfil de egresado al que la universidad aspira.

Este perfil de egresado a su vez, debe estar determinado por las necesidades que se manifiestan en el campo laboral y social, ya que los cambios de la sociedad influyen el aspecto laboral, lo cual genera nuevas problemáticas que requieren de profesionales competentes para solucionarlas. Es esta una de las posturas que debe asumir la universidad, un diseño curricular que permita formar profesionales capacitados para resolver los problemas de la sociedad actual.

Un primer aspecto a considerar en este proceso, es que como universidad determinemos "qué acepción de competencias estaremos utilizando". Con frecuencia, hay acepciones que no son respuesta a los desafíos que enfrenta la educación, porque de alguna manera representan una comprensión empobrecida y confusa del concepto de competencia y responden a planteamientos de corte tecnocrático, aparentemente asépticos, que desprecian o ignoran la dimensión ideológica y ética de la educación (Coll, 2009).

Por su lado, Díaz Barriga (2011, p.3) en su análisis comparativo de los enfoques sobre las acepciones de competencias, indica la importancia de reconocer en dónde estamos posicionándonos a la hora de elegir un significado ya que, según el autor, esto tiene:

implicaciones en quienes formulan las propuestas curriculares, pero también tiene amplia significación en la manera en que los docentes puedan interpretar lo que realizan en el salón de clases. La tensión entre saberes y resolución de problemas, entre calificaciones o expresiones procesuales del avance del estudiante.

He aquí una alerta que debemos tener en las universidades, debido a que, dependiendo de la acepción de competencias asumida, estas pueden implicar un retroceso y/o estancamiento o por el contrario impulsar prácticas realmente innovadoras, susceptibles de mejorar sustancialmente la educación. Las acepciones sobre competencias que pueden incidir en la mejora de la educación, son las que priorizan la integración de diferentes tipos de conocimientos y recursos psicosociales.

Para el desarrollo de estas competencias, el docente debe valerse de contextos reales y complejos que resalten la ejecución competente en el marco de prácticas sociales o socio-profesionales relevantes a cada contexto profesional. Por su parte, las metodologías asociadas con el desarrollo de competencias así entendidas, están basadas en la indagación, como por ejemplo, aprendizaje basado en casos, problemas o proyectos; así también, estas propuestas involucran integración no solo de conocimientos, sino de disciplinas mediante enfoques multi, inter o transdisciplinarios.

En las últimas décadas las reformas educativas de inspiración constructivista han incorporado enfoques curriculares basados simultáneamente en capacidades y contenidos, por lo que esta acepción integradora psicosocial representa un verdadero avance respecto a aquellas.

Hay otras acepciones que no representan avance y, además, dan continuidad a la fragmentación de contenidos curriculares, como son las de inspiración cognitivas o cognitivas conductual y que aún continúan vigentes. Incluso existen acepciones que representan un retroceso, son aquellas que se encuentran con frecuencia y que tienen una visión de las competencias próxima a los planteamientos conductistas o neoconductistas.

Por ello, una responsabilidad de las instituciones de educación superior al respecto, debería consistir en asumir una acepción que represente un avance en materia educativa, definir cómo vamos a entender las competencias, cuáles son sus componentes y cómo se vinculan con el resto de elementos del currículo (contenidos, metodología didáctica, evaluación, etc.). Es decir, definir los alcances teórico-prácticos en las implicaciones curriculares, didácticas y de evaluación para todo el proceso, un verdadero reto de gestión para las universidades.

\section{Competencias}

El Proyecto de Definición y Selección de Competencias (DeSeCo, 2002) de la Organización para la Cooperación y el Desarrollo Económico (OCDE) dice:

Una competencia es la capacidad para responder a las exigencias personales o sociales o para realizar 
una actividad o tarea. Cada competencia reposa sobre una combinación de habilidades prácticas y cognitivas interrelacionadas, conocimientos (incluyendo el conocimiento tácito) motivación, valores, actitudes, emociones y otros elementos sociales y comportamentales que pueden ser movilizados conjuntamente para actuar de manera eficaz (p.18).

Otro aspecto que como universidad se debe asumir, consiste en la necesidad de replantear de manera participativa la organización de los contenidos, para construir sobre núcleos problemáticos, alrededor de los cuales se deben integrar las disciplinas.

Así también es necesario definir, cómo se llevarán a cabo los procesos de evaluación, tan diferentes de los tradicionales, cómo se van a articular los programas de formación docente, la creación de unidades técnicas especializadas y las demás instancias institucionales. Esto implica que la estructura curricular tiene que ser renovada completamente.

Por otra parte, un currículo por competencias, tiene implicaciones no sólo en la reorganización de los contenidos, sino que además cada docente tiene que asumir un compromiso ético con el estudiante, y una actitud que favorezca esa reconstrucción curricular porque tenemos que aprender a trabajar de otra manera.

He aquí otra cuestión a considerar, el currículo es ejecutado por docentes que con frecuencia desconocen su conceptualización pedagógica y la fundamentación epistemológica que lo justifica, con la dificultad adicional de tener que integrar conocimientos de diversas disciplinas, lo cual implica un ejercicio profesional en equipos docentes, si el interés es trabajar por competencias.

Con frecuencia no sólo los docentes desconocen qué implica trabajar por competencias, aunque el diseño curricular así lo exprese. Esto puede deberse a que muchas de las instituciones no tienen ni el conocimiento, ni las condiciones para trabajar por competencias.

Queda claro que un diseño curricular con tales características, desde su planificación, tienen que involucrar a todos los actores y requiere de manera constante programas de capacitación, que prevean espacios de reflexión y análisis en los que se puedan compartir las experiencias y se desarrolle el aprendizaje de cómo trabajar con este enfoque.
A su vez, la capacitación docente no es completa, si carece de investigación de lo que ocurre en el salón y/o de programas de acompañamiento.

...la incorporación de las competencias genéricas en educación superior requiere que los docentes se actualicen en metodologías de enseñanza y evaluación, incorporen actividades prácticas donde observen el despliegue de las competencias esperadas en la asignatura, cuenten con mayor tiempo de preparación de clases, construyan evaluaciones auténticas y entreguen retroalimentación permanente a los estudiantes. Esto involucra una mayor inversión y recursos por parte de las instituciones educativas (Villarroel \& Bruna 2014, p.31).

Un rasgo que caracteriza las competencias y que diferencia significativamente la forma de llevar a cabo el proceso enseñanza aprendizaje, es que la práctica delimita la teoría, porque su núcleo central apunta al "saber hacer" y los demás saberes se agrupan a su alrededor.

Todo lo cual lleva a concluir, que un diseño por competencias debería consistir en la construcción de un currículo pensado-practicado que respeta las subjetividades e incluye sectores y temas invisibilizados, lo que es equivalente a construir un currículo humanizado que fomente una cultura de respeto, para quienes piensan y actúan de manera similar y para el otro que aprende de una manera diferente, piensa de una manera diferente, para el que vive de una manera diferente. Un currículo así pensado, aporta principios e indica caminos que se contraponen a la lógica binaria y supera la concepción rígida del aprendizaje.

Para lograrlo, deberíamos ser capaces, como universidad, de intensificar la discusión académica y respetar el disenso como rasgo que la caracteriza.

Este es el escenario que se presta para que la comunidad académica pueda revisarse y contrastar con la realidad respecto a temas relevantes como igualdad de género, racismo, impacto medio ambiental, desigualdades, etc... con vista a dar respuesta como institución educativa a las necesidades de la sociedad. Además es el espacio para preparar a la juventud a enfrentar un mercado laboral que demanda competencias éticas, de liderazgo, de transparencia y de trabajo en equipo.

Vale decir, diseñar un currículo más realista, más ligado con la naturaleza de la vida, de manera 
que los profesionales estén caracterizados por ser ciudadanos íntegros, líderes emprendedores con alto componente humanístico, preocupados por el mejoramiento personal, del entorno y el bien común.

\section{La gran pregunta es ¿cómo formar para un futuro cambiante?}

Coll (2007, p. 36) al mencionar algunas características por las que pueden resultar ventajosos estos enfoques por competencias, hace referencia a un tipo especial de competencias,

[El diseño de las competencias] que convierten a un aprendiz, en un aprendiz competente, son las que están en la base de la capacidad para seguir aprendiendo a lo largo de la vida, las que permiten desarrollar las capacidades metacognitivas que hacen posible un aprendizaje autónomo y autodirigido. Un aprendiz competente es el que conoce y regula sus propios procesos de aprendizaje, tanto desde el punto de vista cognitivo como emocional, y puede hacer un uso estratégico de sus conocimientos, ajustándolos a las exigencias del contenido o tarea de aprendizaje y a las características de la situación (Bruer, 1995. En Coll 2007).

La selección de los contenidos representa un componente indudablemente ideológico, y además es necesario asumir que esos contenidos tienen que ser apropiados por el estudiante para luego aplicarlos. La toma en consideración de los saberes asociados a las competencias no es sólo una necesidad para asegurar su adquisición y desarrollo, sino que es también una garantía para hacer compatibles dos aspiraciones irrenunciables en el mundo actual: la de educar para el ejercicio de una "ciudadanía universal" y la de educar para el ejercicio de una ciudadanía enraizada en la realidad social, cultural, nacional y regional de la que forma parte (Coll, 2007).

Aunque en algunas propuestas no se visualiza, parece obvio que el contexto requiere ser considerado como elemento fundamental en la selección de contenidos y su implementación, tanto el contexto de donde es adquirida la competencia, como en el que se aplicará, con la intención de que a la vez posibilite que algunos de estos actos intencionales sean generalizables y transferibles (López, 2007).

Si revisamos el análisis de los componentes de competencias dada por USAID (2009) también allí se enfatiza la integración y el contexto, así como el hecho de que, para cumplir con los requisitos de una competencia, no puede faltar ningún elemento, todos tienen que estar presentes y de manera armónica contribuir a un Saber, Saber ser, Saber hacer y Saber ser con los demás. Existe incluso quienes otorgan una concepción de integralidad a las competencias, y reconocen en ellas una función esencial de conjunto, para que los elementos del conocimiento tengan sentido. (Huerta, Pérez García \& Castellanos, 2005).

Al revisar las implicaciones de este tema tan complejo, y para que no pase a ser una moda más en educación, el eje central alrededor del cual tendría que construirse todo currículo por competencias, tiene que ser el ser humano que recibirá la propuesta formativa y su contexto. Ese ser humano que habita en un universo con otros seres vivos, que tiene intereses, sueños, ilusiones, creencias, problemas, amigos, familia, que requiere una formación integral, que no descuide ninguna de sus facetas, capaz de incorporar además de la integración de conocimientos disciplinares, otros saberes no académicos, como arte, cultura, espiritualidad, etc.

En el enfoque del currículo por competencias se hace presente la idea de la formación integral del ser humano, contextualizado, y representaría un importante avance en el diseño curricular, asumir que se diseñen planes y programas pensados alrededor de su bienestar y satisfacción personal, porque si la sociedad no cuenta con seres humanos felices, sus frustraciones, "incompletudes" y sinsabores se verán reflejados en su desempeño profesional por más competentes que sean para tal o cual tarea. Un mundo mejor es posible, con ciudadanos más seguros de sí mismos, más satisfechos con su papel en el mundo.

\section{Conclusión}

No tenemos una solución mágica en educación para la complejidad del mundo actual, sin embargo, el asumir una postura crítica, responsable y participativa quizá puede ayudar al aprovechamiento de lo mejor de cualquier enfoque, en este caso, por competencias.

Por su parte, las competencias para la vida constituyen un eje central si queremos la implementación de este diseño. Pasan en este sentido a ser fundamentales, el aprender cómo aprender, el aprendizaje contextualizado y significativo, la metacognición, adaptación al cambio, reconocerse a sí mismo, reconocer al otro, reconocer el contexto y transformarse en la acción, entre otras. (Tejada, 2006).

De acuerdo al pedagogo chileno Luis Córdova, el avance de la tecnología es apabullante y el profesorado 
ha disminuido impresionantemente en su calidad humanística y cultural. Porque "la calidad primaria del sistema educativo es axiológica, ética, valórica" (...)

Continúa diciendo que los docentes tienen que lograr motivar a los estudiantes más que las computadoras y que un sistema educativo es de calidad, siempre y cuando sea capaz de formar buenos seres humanos (Córdoba, 2015).

Finalmente, no todas las instituciones de educación superior están preparadas para asumir un enfoque por competencias que represente una mejora a lo que teníamos anteriormente. Para que lo estén, se hace necesario seleccionar una acepción de competencias que agregue valor a las propuestas tradicionales, que preste atención a la contextualización de contenidos y sujetos, que la selección y reorganización de contenidos esté claramente definida y responda a los requerimientos de las necesidades de la sociedad y de los actores del hecho educativo. Vale decir, atender la pertinencia y relevancia de ese currículo.

"Me pregunto si las estrellas se iluminan con el fin de que algún día, cada uno pueda encontrar la suya." El Principito. Antoine Saint Exupery

\section{Referencias}

Bruer, J.T. (1995). Escuelas para pensar. Una ciencia del aprendizaje en el aula. Barcelona: Paidós.

Coll, C. (2007). Las competencias en la educación escolar: algo más que una moda y mucho menos que un remedio. Aula de Innovación Educativa, 161, 34-39.

Coll, C. (2009). Los enfoques curriculares basados en competencias y el sentido de aprendizaje escolar. Congreso Mexicano de Investigación Educativa. COMIE. Veracruz, Conferencia magistral. Recuperado en: http://www.psyed.edu.es/ prodGrintie/conf/CC_COMIE09.pdf

Córdoba, M. (noviembre, 2015). Ser docente, una invitación a transgredir, a crear y a soñar. En W. Vélez (Coordinador), VI Simposio Internacional de Estudios Generales. Simposio llevado a cabo en la Universidad Simón Bolívar, Caracas, Venezuela. Recuperado en: http://www.rideg. org/wp-content/uploads/2015/03/Ser-docenteuna-invitaci\%C3\%B3n-a-transgredir-a-crear-y-aso $\%$ C3\%B1ar.pdf
Díaz Barriga, A. (2011). Competencias en educación. Corrientes de pensamiento e implicaciones para el currículo y el trabajo en el aula. Revista Ries. Nro. 5, Vol. II, pp 3-24

Huerta, J., Pérez García, I., Castellanos, A: (2005) Desarrollo curricular por competencias profesionales integrales. Revista Educar, №35, Sección Educación, Oct-dic 2005. Guadalajara, Jalisco, México.

López, A. Sena, I. Javier, J. (26 de junio de 2012). Los efectos de la globalización en la educación. El profesor. Recuperado en: https:// movimiento30juniord.wordpress.com/2012/06/26/ los-efectos-de-la-globalizacion-en-la-educacion/

López Mendoza, T. (Noviembre, 2007) Modelo curricular $y$ las competencias. Mesa de trabajo presentada en el VIII Foro Regional Andino para el Diálogo y la Integración de la Educación Agropecuaria y Rural, Lima, Perú. Recuperado de: http://www. sihca.org/reuniones/todas/Octavoforo/Mesas de_trabajo/1.\%20Desarrollo\%20curricular/4.\%20 UCLA-\%20Tibisay\%20Lopez.doc

Organización para la Cooperación y el Desarrollo Económico (OCDE) (2002) Proyecto de Definición y Selección de Competencias Claves (DeSeCo). Recuperado en: http://deseco.ch/bfs/deseco/en/ index/03/02. parsys.78532. downloadList.94248. DownloadFile.tmp/2005.dscexecutivesummary. sp.pdf

Otero, M. (15 de junio de 2014). Primer paso: formar maestros de excelencia. Períodico La voz del interior. Recuperado en: http://www.lavoz.com.ar/ temas/primer-paso-formar-maestros-de-excelencia

Pinar, W. (2014). La teoría del curriculum. México. Narcea.

Tejada Zabaleta, a. (2006) Diseño curricular basado en competencias para formar profesionales integrales. Universidad del Valle. Colombia

USAID (2009). Guía para la elaboración de sílabo por competencias. USAID: Perú.

Villarroel, V. \& Bruna, D. (2014). Reflexiones en torno a las competencias genéricas en educación superior: Un desafío pendiente. Psicoperspectivas. Vol.13 No.1. pp 23-34 